\title{
Gerstmann's Syndrome: A Rare Clinical Condition with a Tetrad of Symptoms
}

\author{
Virendra C. Patil ${ }^{1}$, Akshay R. Kulkarni ${ }^{2}$ \\ ${ }^{1}$ Professor \& HOD, Department of Medicine, ${ }^{2} J$ unior Resident, Department of Medicine, Krishna Institute of Medical Sciences, \\ Deemed to be University Karad. 415110., India
}

Corresponding author: Dr. Virendra C. Patil, Professor \& HOD, Department of Medicine, Krishna Institute of Medical Sciences, Deemed to be University, Karad. 415110, India

DOI: http://dx.doi.org/10.21276/ijcmsr.2019.4.2.38

How to cite this article: Virendra C. Patil, Akshay R. Kulkarni. Gerstmann's Syndrome: a rare clinical condition with a tetrad of symptoms. International Journal of Contemporary Medicine Surgery and Radiology. 2019;4(2):B173B175.

\section{A B S T R A C T}

Introduction: Gerstmann's syndrome is a neurological condition with a tetrad of symptoms comprising agraphia, acalculia, right-left disorientation and finger agnosia suggesting a lesion in the angular gyrus of the inferior parietal lobule in the dominant hemisphere.

Case report: Here we are reporting a case of a 52 year old right handed male patient presenting with irrelevant talk and slurring of speech and was found to have all four features of the tetrad described by Gerstmann.

Conclusion: Magnetic resonance imaging (MRI) of brain was suggestive of an acute infarct in parietal and temporal lobe on left side.

Keywords: Gerstmann's Syndrome, Inferior Parietal Lobule, Dominant Hemisphere, Angular Gyrus

\section{INTRODUCTION}

Gerstmann's syndrome (GS) corresponds to a tetrad of symptoms comprising acalculia, agraphia, finger agnosia and right-left disorientation. ${ }^{1}$ In the year 1924 , Gerstmann observed few patients having difficulty in discriminating their own fingers, performing calculations, writing and distinguishing left from right. He claimed that these four symptoms constituted a syndromal entity and a lesion of the dominant parietal lobe was responsible for it. ${ }^{2}$ Gerstmann syndrome is a neuropsychiatric disorder. Characteristic tetrad of symptoms suggest the presence of a lesion in the dominant hemisphere involving the inferior parietal lobule. We are reporting a case of Gerstmann syndrome in a 52 years old male.

\section{CASE REPORT}

A 52 years old male patient was brought by relatives and presented with complaints of sudden onset irrelevant talk and slurring of speech since 2 days. Patient had difficulty in understanding written and spoken language. Patient also had difficulty in recalling the exact names of the relatives and objects around him. Fluency of the speech was apparently preserved well. There was no history of obvious limb weakness, loss of consciousness and seizure and fever. On admission patient was afebrile and vitals were stable. On neurological examination, patient had inability in naming familiar objects (anomia), talking around the target word (circumlocution) as well as using entirely new words (neologism). Patient had difficulty in writing (agraphia/ dysgraphia), performing

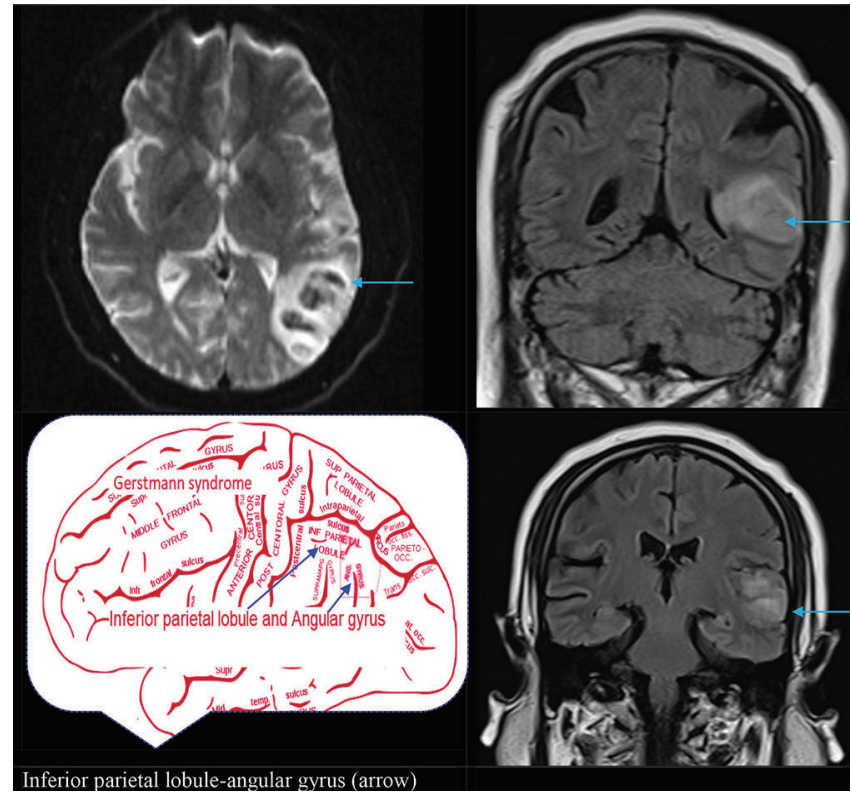

Figure-1: MRI brain shows acute infarct in parietal (inferior parietal lobule angular gyrus), temporal lobe, centrum semiovale and corpus callosum on left side

mathematic calculation (dyscalculia) and left-right disorientation. The patient had finger agnosia for his own fingers and others. Rest of the neurological examination and other system were apparently normal. The laboratory parameters at the time of admission were within acceptable limits except raised blood sugar level. (Table 1)

Patient underwent MRI brain imaging which was suggestive 
of acute infarct in parietal (inferior parietal lobule-angular gyrus) and temporal lobe, centrum semiovale on left side

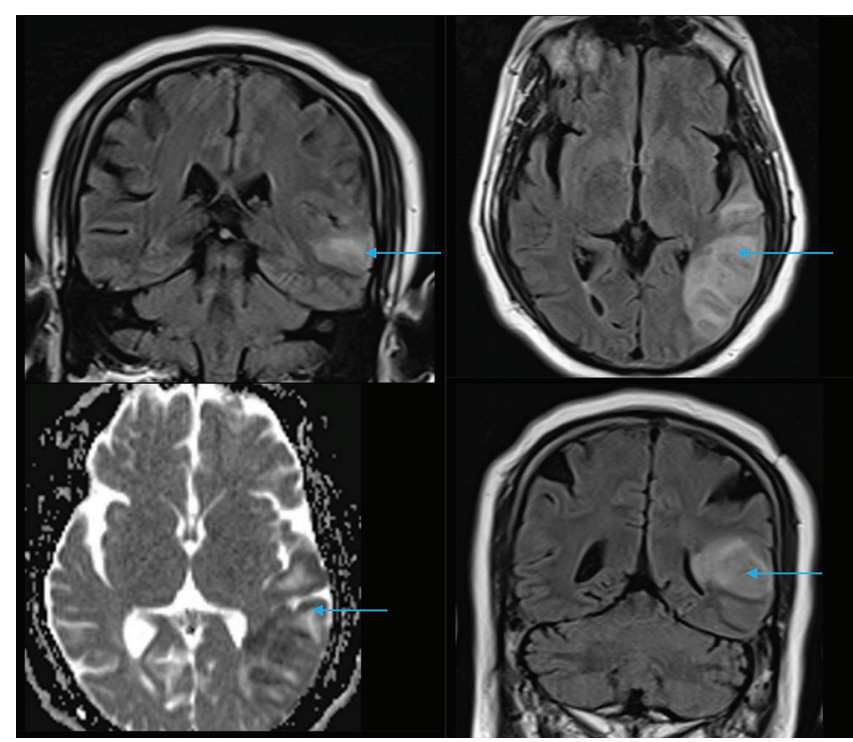

Figure-2: MRI Brain shows acute infarct in parietal and temporal lobe on left side

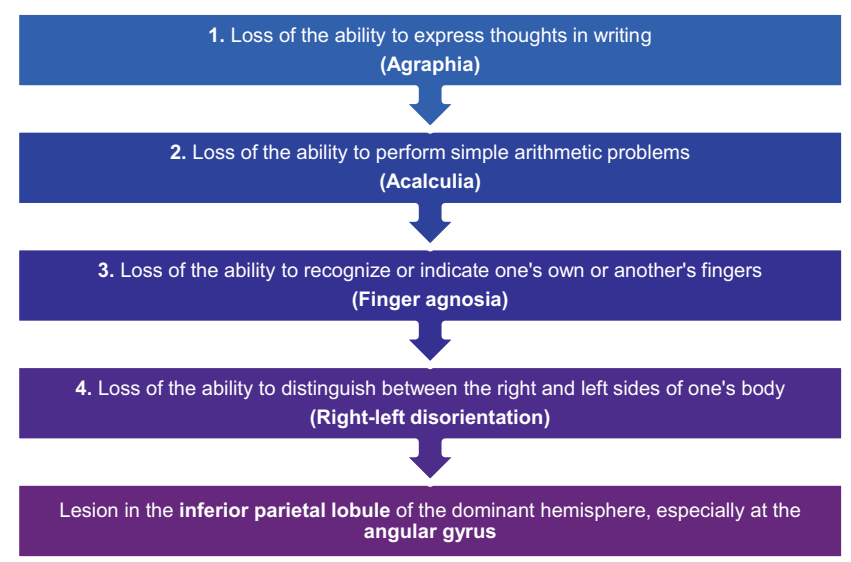

Figure-3: Tetrad of symptoms of Gerstmann syndrome

\begin{tabular}{|l|c|}
\hline Parameters & Value \\
\hline Haemoglobin & $12.5 \mathrm{gm} / \mathrm{dl}$ \\
\hline Total Leukocyte count & $11300 / \mathrm{cmm}$ \\
\hline Platelet count & $1.90 / \mathrm{cmm}$ \\
\hline $\begin{array}{l}\text { Prothrombin time / International Normalised } \\
\text { Ratio }\end{array}$ & $12.1 / 1.1$ \\
\hline Urine examination & $\mathrm{WNL}$ \\
\hline HbA1c & $6.7 \%$ \\
\hline Random blood sugar & $171 \mathrm{mg} / \mathrm{dl}$ \\
\hline Blood urea & $21 \mathrm{mg} / \mathrm{dl}$ \\
\hline Serum creatinine & $0.87 \mathrm{mg} / \mathrm{dl}$ \\
\hline Serum Sodium & $137 \mathrm{mEq} / \mathrm{L}$ \\
\hline Serum potassium & $3.9 \mathrm{mEq} / \mathrm{L}$ \\
\hline Liver Function tests & Normal \\
\hline Serum Albumin & $3.9 \mathrm{~g} / \mathrm{dl}$ \\
\hline Lipid profile & WNL \\
\hline USG A/P & WNL \\
\hline Carotid Doppler study & Normal \\
\hline \multicolumn{1}{|c|}{ Table-1: Laboratory parameters at time of admission } \\
\hline
\end{tabular}

and corpus callosum (figure $1 \& 2$ ). Patient was diagnosed to have left middle cerebral artery territory infarct with features suggestive of Gerstmann syndrome (tetrad of symptoms comprising agraphia, acalculia, right-left disorientation and finger agnosia (Figure-3). The patient was treated with antiplatelets, statins, antihypertensive, oral hypoglycaemic agent and other supportive medications. The patient showed partial improvement in the form of improvement in the following commands, reduced finger agnosia and rightleft disorientation. On discharge patient was prescribed antihypertensive, dual antiplatelet and oral hypoglycaemic agent.

\section{DISCUSSION}

Joseph Gerstmann was an Austrian neurologist. In 1924 he described the case of a 53 year

old patient presenting with difficulty in writing, performing simple mathematical

calculations, right-left disorientation and difficulty in moving specific fingers when requested

by the examiner. All these clinical findings were related to cerebrovascular syndrome with left hemisphere involvement. ${ }^{1}$ Three years later, Gerstmann reported two similar cases exhibiting difficulty reading numbers but not words, constructional apraxia and anomia for colours. ${ }^{2}$ Gerstmann's work was published in English in the year 1940 and then the syndrome became known globally. Gerstmann syndrome was characterized by the tetrad comprising: agraphia, acalculia, finger agnosia and right-left disorientation. In present case, all four features described by Gerstmann were present. In the following decades, a strong relationship between lesions in the dominant hemisphere and Gerstmann syndrome was confirmed, more specifically in the angular gyrus of the parietal lobe..$^{3-7}$ The lesions in this gyrus are associated predominantly with the symptoms of agraphia and acalculia. ${ }^{8}$ MRI brain of the patient in present case was suggestive of involvement of parietal lobe of dominant hemisphere. Ardila $\mathrm{A}$ et al in their article'A proposed reinterpretation of Gerstmann's syndrome' in 2014 suggested that semantic aphasia is always associated with acalculia; as a matter of fact, left angular gyrus has a significant involvement in semantic processing.

Gerstmann's syndrome should include: acalculia, finger agnosia, right-left disorientation and semantic aphasia, but not agraphia. When the pathology extends toward the superior parietal gyrus, agraphia can be found. ${ }^{9}$ In present patient, we didn't find semantic aphasia. Zukic S et al analysed 194 acute stroke patients and found to have 59 $(30.40 \%)$ with alexia, agraphia and acalculia or different combinations of these disorders. Two patients (3.4\%) had agraphia and acalculia associated with features of Gerstmann syndrome. The computed tomography scan in these cases showed ischemic lesion in the left parietal and left temporoparietal lobe i.e. angular gyrus of the dominant hemisphere. ${ }^{3}$ MRI brain of the patient in present case was also suggestive of involvement of left parietal lobe. In many cases of lesion to the left frontal lobe, features of Gerstmann syndrome have been reported but none of these fulfilled all four diagnostic criteria. Study done by Eun-Ju Lee et al reported two cases 
with tetrad of Gerstmann's syndrome with lesion involving the left middle frontal lobe, sparing the angular gyrus. Disconnection of functional fibres between the frontal and parietal cortex forms the basis for the possible explanation of this discrepancy. Left frontal cortex or the subcortical area are important functional areas, closely connected with the parietal lobe in Gerstmann's syndrome. ${ }^{10}$ In present case we didn't find any involvement of frontal lobe on MRI brain. Bhattacharyya $\mathrm{S}$ et al reported a 68-year right handed woman with sudden onset inability in completing a Sudoku grid and was found to have dyscalculia, dysgraphia and left-right confusion. Magnetic resonance imaging (MRI) showed the acute infarct in the left posterior insula and temporo-parietal operculum..$^{11}$ In adults, stroke or other insult to the brain can give rise to the syndrome. The parietal lobes concerned with perception of sensation and understanding the sensory input, get involved in Gerstmann syndrome.

\section{CONCLUSION}

Characteristics of the Gerstmann syndrome include loss or absence of four cognitive functions- the loss of the ability to express thoughts in writing (agraphia, dysgraphia), to recognize one's own or another's fingers (finger agnosia), to perform simple arithmetic problems (acalculia) and to distinguish between the right and left sides of one's body. Gerstmann syndrome though included in a rare syndrome, is an important neurological condition not to be missed. The lesion in the brain mainly localized to the angular gyrus of the dominant hemisphere. Thus presence of Gerstmann syndrome has got significant predictive value in localising cerebral hemispheric lesion.

\section{REFERENCES}

1. Rafael Batista João, Raquel Mattos Filgueiras, Marcelo Lucci Mussi, and João Eliezer Ferri de Barros Dement. Transient Gerstmann syndrome as manifestation of stroke: Case report and brief literature review. Neuro psychol. 2017;11(2):202-205.

2. Gerstmann J. Fingeragnosie and Agraphie-einneues Syndrom. Arch Psychiatr Nervenkr Z Gesamte Neurol Psychiatr. 1927;108(2):152-177.

3. Zukic S, Mrkonjic Z, Sinanovic O, Vidovic M, Kojic B. Acta. Gerstmann'S syndrome in acute stroke patients. Inform Med. 2012;20(4):242-3.

4. Mayer-Gross W. Some observations on apraxia. Proc R Soc Med. 1935;28(6):1203-1212.

5. Roeltgen DP, Sevush S, Heilman KM.Pure Gerstmann's syndrome. Arch Neurol. 1983;40(6):46-47.

6. Mazzoni M, Pardossi L, Cantini R, Giorgetti V, Arena R. Gerstmann syndrome: a case report. Cortex. 1990;26(8):459-467.

7. Tucha O, Steup A, Smely C, Lange KW. Toe agnosia in Gerstmann syndrome. J Neurol Neurosurg Psychiatry. 1997;63(5):399-403.

8. Greenblatt SH. Alexia without agraphia or hemianopsia. Anatomical analysis of an autopsied case. Brain. 1973;96(6):307-316

9. Ardila A. A proposed reinterpretation of Gerstmann's syndrome. Arch Clin Neuropsychol. 2014;29(8):828-33.

10. Lee EJ, Shin HY, Noh Y, Park KH, Park HM, et al.
Two Cases with Cerebral Infarction in the Left Middle Frontal Lobe Presented as Gerstmann's Syndrome. J Neurol Disord. 2016;4(8):309.

11. Bhattacharyya S, Cai X, Klein JP. Dyscalculia, dysgraphia, and left-right confusion from a left posterior peri-insular infarct. Behav Neurol. Volume 2014, Article ID 823591, 4 pages.

\section{Source of Support: Nil; Conflict of Interest: None}

Submitted: 28-02-2019; Accepted: 04-06-2019; Published online: 29-06-2019 